\title{
How playing area dimension and number of players constrain football performance during unbalanced ball possession games
}

International Journal of Sports Science \& Coaching $0(0) 1-10$

(C) The Author(s) 2020

Article reuse guidelines: sagepub.com/journals-permissions DOI: $10.1177 / 1747954120966416$ journals.sagepub.com/home/spo ๑SAGE

\author{
Nuno André Nunes ${ }^{1,2}$ (D), Bruno Gonçalves ${ }^{3,4,5}$, \\ Diogo Coutinho ${ }^{2}$, Fábio Yuzo Nakamura ${ }^{2,6}$ and \\ Bruno Travassos ${ }^{1,2,5}$ (D)
}

\begin{abstract}
The aim of this investigation was to analyse the external workload, tactical individual actions of passing, and perceived internal load during unbalanced small-sided games. Ball possession formats $(4 \mathrm{v} 3,4 \mathrm{v} 4$ and $4 \mathrm{v} 5)$ were played in three different playing area dimensions $(20 \times 15 \mathrm{~m}, 25 \times 20 \mathrm{~m}$ and $30 \times 25 \mathrm{~m})$ by under- 23 football players. Data were analysed under opposition-based perspective, by fixing one team $(4 \mathrm{vX})$, and by cooperation-based perspective according to teammates $(4 \mathrm{v} 2+\mathrm{X})$ for each playing area condition. GPS monitors were used to collect and compute external workloads (distance covered while walking, running, sprinting, and maximal speed) and tactical individual actions (passing with dominant and non-dominant foot, and maximum passing speed), and Borg Scale CRIO to evaluate rating of perceived exertion (RPE). On both opposition- and cooperation-based perspectives, significant differences were found on external workload variables for all game formats, with smaller areas associated with more distances covered while walking and larger areas with running and sprinting. Likewise, $4 \mathrm{v} 3,4 \mathrm{v} 4$ and $4 \mathrm{v} 2+3$ revealed significant differences for tactical individual actions, where a larger area was associated with an increase in repetitions. Medium playing area, for both perspectives, was associated with a higher RPE. Overall, larger playing areas with higher number of players involved promoted more high-intensity running, while the same area with fewer number of players fostered tactical individual actions. Smaller areas allowed to reduce game pace, especially in formats with fewer players. Different unbalance scenarios under dissimilar playing area dimensions promote diverse performance outcomes on player's action capabilities.
\end{abstract}

\section{Keywords}

Constraints-led approach, global positioning system, rating of perceived exertion, small-sided games, soccer

\section{Introduction}

As part of the Constraint-Led Approach (CLA), sports performance and the acquisition of skills emerge from the interaction between the players, the task and the environment. ${ }^{1}$ This approach advocates that the creation of learning environments, such as small-sided games (SSGs), facilitates discovery, guiding the player through a variety of possible movement solutions in the search for an optimal response to ever-changing environment. ${ }^{2}$ Thus, coaches can manipulate the training environment using task constraints, which are specific variables used to create specific contexts and stimulate players' behaviour for a given result, attracting them to
Reviewer: Carlos Lago-Peñas (University of Vigo, Spain)

'Department of Sports Sciences, University of Beira Interior, Covilhã, Portugal

${ }^{2}$ Research Centre in Sports Sciences, Health, Sciences and Human Development, CIDESD, CreativeLab Research Community, Vila Real, Portugal

${ }^{3}$ Departamento de Desporto e Saúde, Escola de Ciências e Tecnologia, Universidade de Évora, Évora, Portugal

${ }^{4}$ Comprehensive Health Research Centre (CHRC), Universidade de Évora, Évora, Portugal

${ }^{5}$ Portugal Football School, Portuguese Football Federation, Oeiras, Portugal

${ }^{6}$ Associate Graduate Program in Physical Education UPE/UFPB, João

Pessoa, Brazil

\section{Corresponding author:}

Nuno André Nunes, University of Beira Interior - Department of Sports Science, Convento de Sto. António, 6201-00I Covilhã, Portugal.

Email: nunoandrenunes@live.com.pt 
an explicit action and expanding a range of information sources. ${ }^{3,4}$

Manipulating the number of players on task is commonly one of the constraints used by coaches on a daily basis. ${ }^{5}$ Accordingly, the coordination processes within and between players and their action capabilities while performing important dynamic interceptive actions and order-disorder transitions can destabilize or (re)stabilize the game, being related with the number of teammates and opponents on task. ${ }^{2}$ However, to date, most of the studies have been focusing on game conditions where both teams are in numerical equality, even though soccer is often played in unbalanced numbers per side, either momentary or permanently. ${ }^{6,7}$ Some of the studies carried out on unbalanced scenarios have reported that the variation of the players' number has a greater influence on the external load and RPE than on the physiological responses; although the team in numerical inferiority perceives the task as more intense compared with the team in superiority, there are no significant differences in the external load and physiological responses. ${ }^{8}$ Likewise, Vilar, Araújo ${ }^{9}$ demonstrated the importance of numerical advantage as a key element in maintaining defensive stability and creating opportunities for success in the offensive phase. Meanwhile, Sampaio, Lago ${ }^{10}$ suggested that playing with a numerical disadvantage decreases the randomness of the players' distances to the center of the team, promoting more predictable behaviors.

Also, it was observed that the manipulation of playing dimensions in balanced numerical relationships constraints the intensity of the game, the actions of the players and the energy pathways used. ${ }^{11}$ More specifically, large playing areas are associated with an increase in the intensity of exercise ${ }^{12,13}$ and effective playing space and surface coverage, ${ }^{14}$ while small playing areas appear to foster technical development. ${ }^{15}$ When manipulating both number and playing area dimension constraints concurrently, it was noted that the total distance and the distance covered at higher intensities, as well as maximum speed, acceleration and deceleration increased with the addition of players and a larger playing area $(10 \mathrm{v} 10>7 \mathrm{v} 7>5 \mathrm{v} 5)$; on the contrary, the number of accelerations and decelerations, as well as the total number of changes in speed, were higher as the dimensions of the playing area and the number of players decreased $(5 \mathrm{v} 5>7 \mathrm{v} 7>10 \mathrm{v} 10){ }^{16}$ However, to the best of our knowledge, no studies have been conducted to analyse the effects of the playing area manipulation on different unbalanced formats. It remains unclear how players behave in different game scenarios when confronting unbalanced number of opponents or adapting to teammates behaviours in different playing areas.
Specially, it is important to understand the effects of the aforementioned conditions on ball possession scenarios, as these drills are widely used by coaches to develop combined game skills. ${ }^{17}$ In these tasks, the generic objective is to keep ball possession without any goal or goalkeeper involved and it can promote an increase in exercise intensity and technical requirements, as players have to move faster to maintain ball possession, as opposed to the use of goals where players adjust behaviours to score faster. ${ }^{15}$ Adopting the constraint of maintaining ball possession, the offensive processes were characterised by increasing the number of passes and the number of players involved in the actions. ${ }^{18}$ This task constraint elicits greater physical and physiological responses on players, and varying the number seems not to produce significant differences on physiological responses, but reduces physical workload as the number decreases. ${ }^{19}$ Therefore, ball possession SSGs revealed to be more intense as players need to constantly create passing lines or to block opponents, thereby increasing the number of moves. ${ }^{18}$

Hence, the aim of this study was to evaluate the effects of the unbalanced number of players $(4 \mathrm{v} 3,4 \mathrm{v} 4$ and $4 \mathrm{v} 5$ ) in ball possession SSGs on under-23 football players' performance, in three different playing areas (small: 20x15m; medium: $25 \times 20 \mathrm{~m}$ : and large: $30 \times 25 \mathrm{~m}$ ), under perspectives of opposition and cooperation. In opposition, it is expected that, as higher the number of players involved in the task, the lower the internal and external load. ${ }^{7}$ From a cooperation perspective, it is expected higher values of internal and external workload for players in situations of numerical inferiority, especially in lower number formats. ${ }^{20}$ Also, for both perspectives, according to the theoretical principles of ecological dynamics, larger playing areas are expected to benefit players seeking to maintain ball possession; from the players seeking to recover ball possession, it is expected that they will have fewer opportunities to intercept the ball in larger playing areas due to the increase in interpersonal distances between individuals. ${ }^{21,22}$

\section{Methods}

\section{Participants}

Twenty under-23 university-level football players, playing at a semi-professional level, participated in this study (age $22.3 \pm 2.0$ years, body mass: $71.4 \pm 7.0 \mathrm{~kg}$, height: $177.1 \pm 6.8 \mathrm{~cm}$, years of experience: $12.1 \pm$ 3.7 years). All participants were part of the same team and experienced three weekly 90 -minute training sessions, plus one game on weekends at a regional playing standard in a regular turf football pitch. Players have around 40 weeks of training per sporting season and 
goalkeepers were not included in data collection. The experimental protocol and investigation were approved by the local Institutional Research Ethics Committee and performed in accordance with the ethical standards of the Helsinki Declaration. Written informed consent procedure was undertaken with all participants, the coach and the club before data collection, when it was also informed the benefits and risks of the investigation.

\section{Procedures}

Participants performed in a series of $4 \mathrm{vX}(4 \mathrm{v} 3,4 \mathrm{v} 4$ and $4 v 5) \mathrm{SSGs}^{23,24}$ aiming at ball possession on different playing area dimensions: small playing area (S: 20x15m), medium playing area (M: $25 \times 20 \mathrm{~m})$ and large playing area $(\mathrm{L}: 30 \times 25 \mathrm{~m})$ on an artificial turf pitch. ${ }^{25,26}$ Head coaches were present during data collection and assigned players into balanced teams, as result of their perception on players' physical, technical, tactical and perceptual capabilities. In the opposition-based perspective, variables were analysed by fixing the same four players and comparing them against 3 (Superiority; Sup, 4v3), 4 (Balanced, 4v4) and 5 (Inferiority; Inf, 4v5) players. In the cooperationbased perspective, variables were analysed by comparing performances from the same 2 players when counting with 1 (Low; LowCoop, 4v2 + 1), 2 (Balanced, $4 \mathrm{v} 2+2$ ) and 3 (High; HighCoop, 4v2 +3) teammates $^{7,27}$ (Figure 1). The different SSGs (4v3, 4v4 and $4 \mathrm{v} 6$ ) were performed for 4 sets of 4 minutes each, with four minutes of active recovery between games (in a total of sixteen minutes of intermittent exercise for each SSG; 4 x $4 \mathrm{~min}+4$ min recovery time). ${ }^{12}$ Three training sessions were completed on 3 different days, with each session being played on the same playing area (day 1 on $\mathrm{M}$; day 2 on $\mathrm{S}$; and day 3 on $\mathrm{L}$ ). Additionally, each training session was performed in the same day of the microcyle, with a week of difference. Before the beginning of each session, players performed a general warm-up that included running at various intensities, joint mobilization and stretching, for 20-min duration. Due to the purpose of the exercise (maintaining and recovering ball possession), no goal or target was used, and a free-play game was adopted, where players could use as many ball touches as desired. The coach did not intervene during the SSG with any type of feedback nor any kind of players' encouragement was provided. If the ball went out of play, other strategically placed balls allowed an immediate restart from a pass.

\section{Data collection}

Data on the external workload variables were collected through a Global Positioning System (GPS) included in the ZEPP Play Soccer system (ZEPP Labs, San Jose, United States), which uses 2 Micro Electromechanical Systems (MEMS) sensors and Bluetooth 4.0 Low Energy (LE) connectivity. Each player had a microchip (each with 2 internal sensors: 3-Axis Accelerometer + 3-Axis Gyroscope) attached to each of their gastrocnemius to record displacement data. $^{28,29}$ Later, Zepp's computer software (version 1.6.0) was used to compute the values of total distance covered $(\mathrm{m})$, differentiated by walking $(\leq 9 \mathrm{~km} / \mathrm{h})$, running $(9-18 \mathrm{~km} / \mathrm{h})$ and sprinting $(>18 \mathrm{~km} / \mathrm{h})$, number of sprints $(\mathrm{n})$, maximum sprint speed $(\mathrm{km} / \mathrm{h})$, number of passing (ball contacts) with dominant and nondominant foot (n), and maximum passing speed $(\mathrm{km} /$ h). ${ }^{30}$ The internal load was assessed using RPE through the Borg's CR10 Scale. It was presented to participants after the end of each SSG bout to ensure that the perceived effort was referred to that specific game. ${ }^{31}$ For the tactical individual actions of passing, it was counted when the force applied to the ball allowed it to travel a distance of at least 5 meters, using ZEPP Play Soccer system.

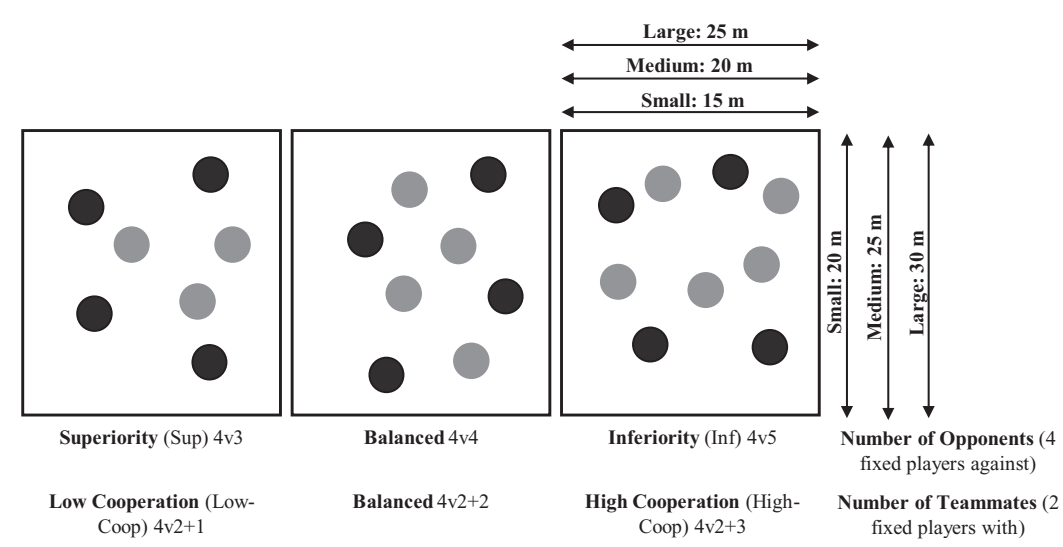

Figure I. Small-sided games design. 


\section{Statistical analysis}

The statistical analysis was completed using The Jamovi Project. ${ }^{32}$ A descriptive analysis was performed with the values of Median, and Min and Max. A Shapiro-Wilk test was used to assess the normal distribution of data. Due to the existence of non-normal distribution of data, the differences between playing areas were assessed using a non-parametric test (Friedman test), combining the different playing areas for each SSG format, with $p$ set at $\leq 0.05$. Additionally, pairwise comparisons were carried according with two scenarios: (i) oppositionbased perspective, where it was analysed the effects of the playing area dimension when manipulating the number of opponents for each game format; and (ii) cooperation-based perspective, where it was analysed the effects of the playing area dimension when changing the number of teammates for each game format. All differences were assessed via standardised differences with pooled variance, derived from the mean and standard deviation of each variable. The magnitude thresholds for effect size of mean differences (Cohen's d) were $0.20,0.60,1.2,2.0$ and 4.0 for small, moderate, large, very large and extremely large, respectively. ${ }^{33,34}$

\section{Results}

\section{Opposition-based perspective}

Table 1 shows descriptive analysis with Median and Min - Max. Table 2 presents Friedman test results with $p$ set at $<0.05$ and Cohen's d effect sizes.

On Sup format $(4 \mathrm{v} 3)$, large effects were found on running $(p<0.01,-1.04)$ and $\operatorname{RPE}(p<0.05,-0.604)$ when comparing $\mathrm{S}$ vs $\mathrm{M}$, and on RPE $(p<0.05,0.664)$ when comparing $\mathrm{M}$ vs $\mathrm{L}$. A higher number of differences with moderate and large effects were found on every external workload variable (walking: $p<0.05,0.487$; running: $p<0.001,-0.899$; sprinting: $p<0.05,-0.745$ and max speed: $p<0.01,-0.826)$, max passing speed $(p=0.05,-0.319)$ and non-dominant foot passes ( $p<0.05,-0.490$ ) when comparing $\mathrm{S}$ vs $\mathrm{L}$ playing areas.

On Balanced format $(4 \mathrm{v} 4)$, walking $(p<0.01,0.787)$, running $(p<0.05,-0.591)$, RPE $(p<0.05-0.893)$ and max passing speed $(p<0.05,-0.400)$ revealed moderate and large effects when comparing $\mathrm{S}$ vs $\mathrm{M}$ playing areas, as well as RPE $(p<0.01,0.866)$ and passing number ( $p=0.05,-0.373$ ) when comparing $\mathrm{M}$ vs $\mathrm{L}$ playing areas. Very large effects were found when comparing $\mathrm{S}$ vs $\mathrm{L}$ playing areas, on walking and running (both $p<0.001,1.25$ and -1.33 , respectively), and moderate effect on sprinting $(p<0.05,-0.533)$, and in every tactical individual action variable (moderate and large effects on max passing speed: $p<0.05,-0.436$; passing number: $p<0.01,-0.769$; dominant foot: $p<0.05$,
-0.702; and non-dominant foot: $p<0.05,-0.641$ ). This balanced number format showed a higher total number of differences when comparing the different playing areas.

For Inf format (4v5), a higher amount of differences was found on the external and internal loads, while no differences were noticed on tactical individual actions. On $4 \mathrm{v} 5$, large effects were found on running ( $p=0.001$, $-0.702)$ and RPE $(p<0.01,-1.03)$ when comparing $\mathrm{S}$ vs $\mathrm{M}$; all external workload variables showed differences (moderate and large reduction effects, with exception of walking) when comparing S vs L (all $p=<.001$ ) and $\mathrm{M}$ vs $\mathrm{L}$ (all $p<0.01$ or 0.001$)$, while $\operatorname{RPE}(p<0.01$, $0.664)$ differed in $\mathrm{M}$ vs $\mathrm{L}$.

\section{Cooperation-based perspective}

Table 1 shows descriptive analysis with Median and Min - Max. Table 2 presents Friedman test results with $p$ set at $<0.05$ and Cohen's d effect sizes.

In the LowCoop format $(4 \mathrm{v} 2+1)$, large effects were found on running ( $p<0.05,-0.826)$, sprinting $(p<0.05$, $-0.833)$ and max speed $(p<0.05,-0.870)$ when comparing $\mathrm{S}$ vs $\mathrm{L}$, and large and moderate effects on walking $(p<0.01,0.753)$ and running $(p<0.05,-0.445)$ when comparing $\mathrm{M}$ vs $\mathrm{L}$ playing areas. No significant differences were found on RPE. Likewise, no differences were found for tactical individual actions.

In the $4 \mathrm{v} 4$, large effects were found on RPE $(p<0.01,-0.828)$ when comparing $\mathrm{S}$ vs $\mathrm{M}$; on sprinting $(p=0.001,-0.820)$, max speed $(p=0.01,-0.632)$ and RPE $(p<0.01,-0.796)$ when comparing $\mathrm{S}$ vs $\mathrm{L}$, and moderate effects on sprinting $(p<0.05,-0.536)$ when comparing $\mathrm{M}$ vs $\mathrm{L}$ playing areas.

The HighCoop $(4 \mathrm{v} 2+3)$ was the format that revealed more effects between playing areas. Moderate effects were found on running $(p<0.01$, -0.362) and max passing speed $(p<0.01,-0.563)$ when comparing $\mathrm{S}$ vs $\mathrm{M}$. Large effects on sprinting $(p=0.001,-0.934)$ and max speed $(p<0.01,-0.757)$ were noted when comparing $M$ vs $L$ playing areas. All external workload variables (moderate and large reductions as detected by effect sizes, with exception of walking) revealed differences when comparing $\mathrm{S}$ vs $\mathrm{L}$ playing areas (all $<0.05,<0.01$ and $<0.001$ ), as well as max passing speed $(p=0.001,-0.857)$ and number of passes $(p<0.01,-0.331)$.

\section{Discussion}

The aim of this study was to evaluate the effects of the playing area dimensions (small, medium and large) during unbalanced number ball possession SSGs (4v3, $4 \mathrm{v} 4$ and $4 \mathrm{v} 5$ ), in opposition- and cooperation-based perspective game conditions, on under- 23 football 


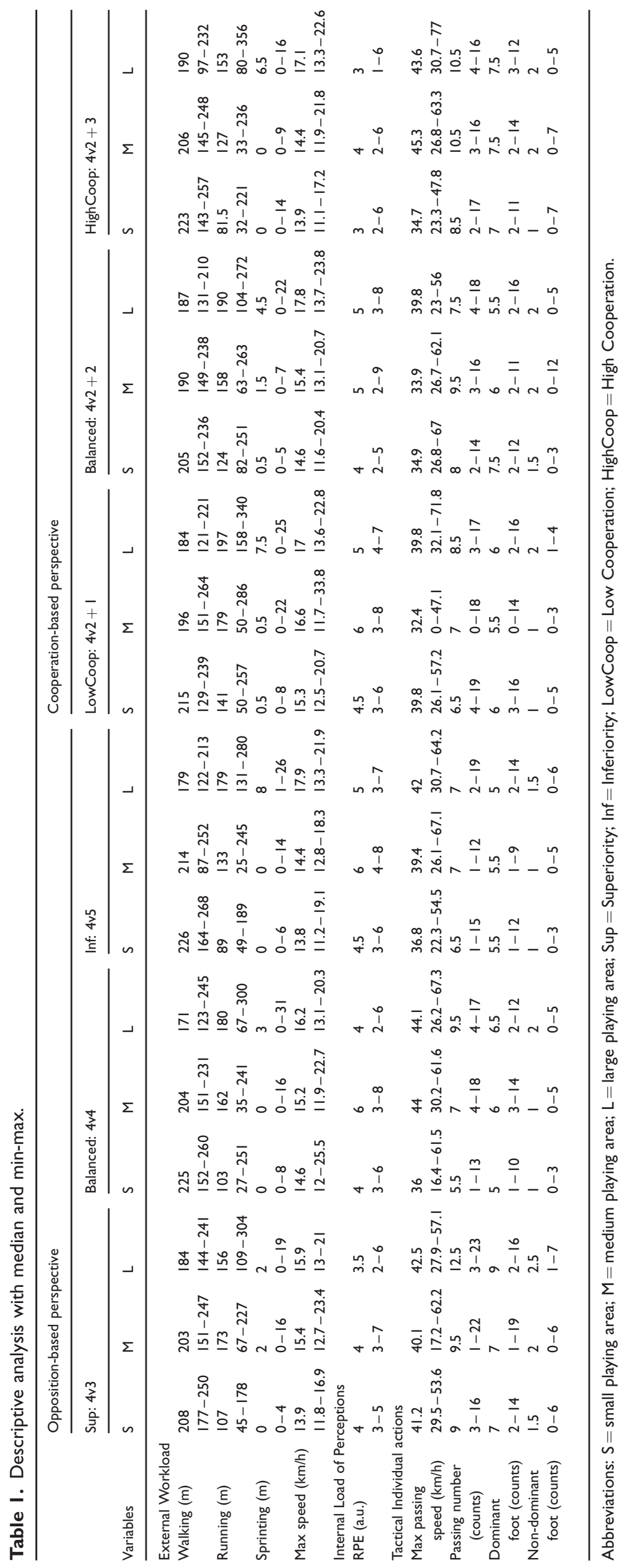




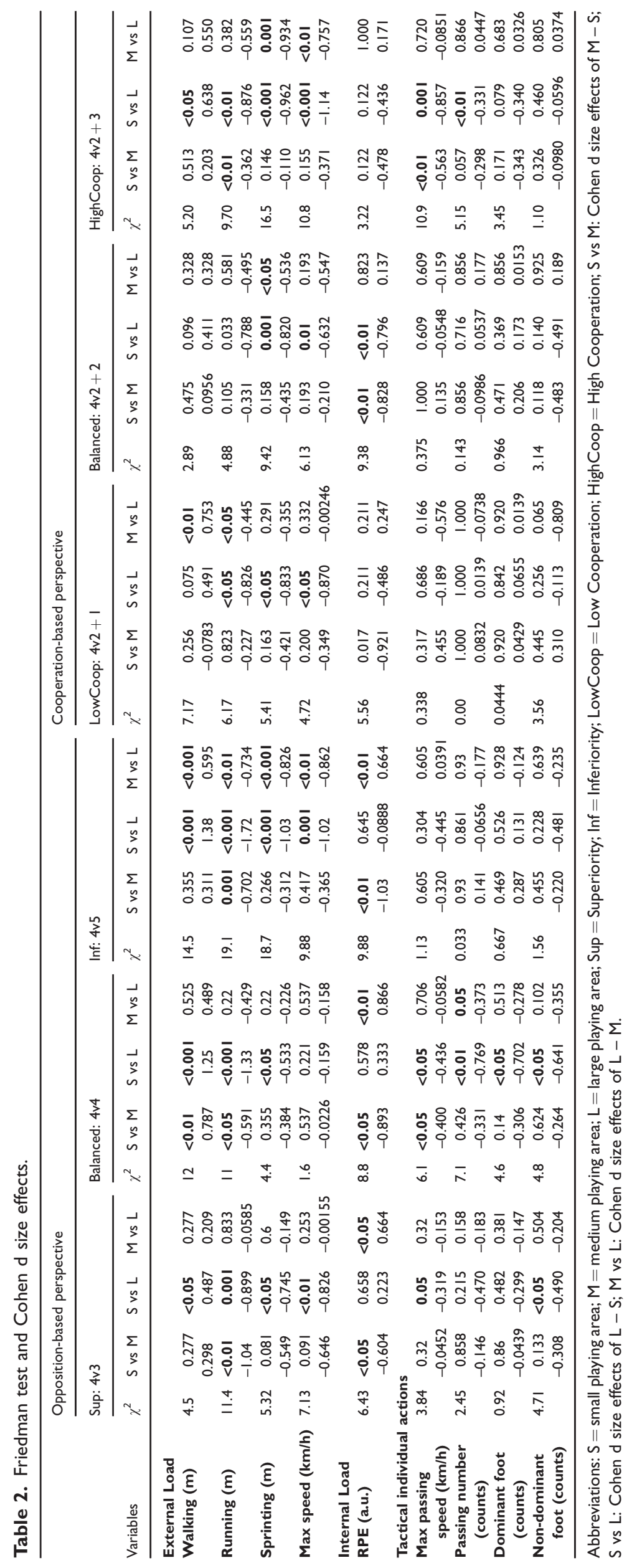


players' performance. Most of the differences were observed in larger areas ( $\mathrm{M}$ and $\mathrm{L}$ playing areas), with higher number of players involved $(4 \mathrm{v} 4$ and $4 \mathrm{v} 5$ formats). Specifically, from an opposition perspective, Balanced formats $(4 \mathrm{v} 4)$ and L playing areas promoted more intensive running and tactical individual actions. From a cooperation perspective, larger areas with higher level of cooperation $(4 \mathrm{v} 2+3)$ allowed more tactical individual actions, while a lower level of cooperation $(4 \mathrm{v} 2+1)$ demanded higher intensity running. For both perspectives, $\mathrm{M}$ playing areas promoted a higher $\mathrm{RPE}$ while $\mathrm{S}$ playing areas allowed more walking and lower game intensity.

\section{Opposition-based perspective}

From an opposition-based perspective, as higher the number of players involved in the task, as more significant differences found in terms of external load. It is at odds of the results of Torres-Ronda, Gonçalves, ${ }^{7}$ who showed that higher number of players involved in the task led to lower internal and external load. These differences may result from the dissimilar rules adopted, as in the mentioned study the authors have used goals, which may constrain the team in inferiority to stay compact to protect the goal, contributing to a lower physical demand. However, in this study, the main aim was to maintain ball possession, which may have triggered the players to move faster to recover the ball, and to block possible passing lines despite their numerical inferiority.

In terms of space manipulation, it is well reported that playing area dimensions influence the intensity of the game, the actions of the players and the energy systems used, ${ }^{11}$ with large playing areas associated with an increase in the intensity of exercise. ${ }^{12,13}$ However, most of the studies analysed the playing area dimension under balanced formats and using goals. Considering the increasing popularity of ball possession tasks in soccer, it is important to understand how different playing area dimensions and superiority/ inferiority relations impacts the players' behaviours to better assist coaches to design sessions. From this perspective, the results showed an increase in the distance covered at higher speeds during the larger formats, which is linked with the higher available space for the players to perform. ${ }^{22}$ Accordingly, in smaller spaces, players are more likely to play close to each other to provide fast and short passing lines. Meanwhile, in larger playing area dimensions, players in possession are more likely to move to free spaces to provide safer passing lines (i.e., further away from the opponents), contributing to an increase in the distance covered at higher speeds. ${ }^{35}$ Furthermore, this increase in the physical demands was more evident when playing against a higher number of opponents (Inf, 4v5). Accordingly, when facing a higher number of opponents, mainly under inferiority situations, it is likely that the team stay less time in possession and more time attempting to press the opponents and recover the ball, contributing to a higher physical demand. In fact, the results from the tactical individual actions seem to support this evidence, as there were less ball contacts when facing a higher number of opponents. Therefore, playing in Inf and in larger playing areas may have constrained the players to develop a team auto-organisation as a compact unit, promoting more intensive movements to recover ball possession, and consequently, spending more energy while trying to keep ball possession. ${ }^{27,36}$

The aforementioned results suggest that coaching staff seeking to improve endurance of the players might adopt $\mathrm{L}$ playing areas using $4 \mathrm{v} 4$ and $4 \mathrm{v} 5$ formats due to the greater running distance covered when compared to smaller playing areas. This likely promotes higher physiological stimulation of the aerobic fitness. ${ }^{37}$ In fact, training time at high intensity is significantly correlated with speed improvement at the lactate threshold speeds $(r=0.84[2 \mathrm{mM}]$ and 0.65 $[4 \mathrm{mM}])$ in elite football players. ${ }^{38}$ In addition, the $4 \mathrm{v} 5$ in $\mathrm{L}$ playing area leads to higher sprinting and Max speed than $\mathrm{S}$ and $\mathrm{M}$, and this can be meaningful to develop anaerobic capacity and speed characteristics, which are often overlooked while designing SSG. ${ }^{39}$ The higher intensities in $4 \mathrm{v} 5$ in comparison to $4 \mathrm{v} 4$ and $4 \mathrm{v} 3$ could be achieved without increasing the corresponding RPE, and this can be relevant to stress the high-speed related abilities without increasing the internal load of the session.

In regard to tactical individual actions, it was also revealed interesting results when manipulating the number of opponents. For instance, a higher number of passing actions were found in Sup (4v3) and Balanced (4v4) formats in $\mathrm{M}$ and $\mathrm{L}$ playing areas, compared to $\mathrm{S}$. That is, when playing against a lower number of opponents, players seem to be able to identify available passing lines without the need to explore all the playing area surface, as a result of having one more player than the opposing team. In turn, when more opponents are added to the task (Balanced, $4 \mathrm{v} 4$ ), players seem to need to explore more their movements in the available playing area as an emergent behaviour to find free space and receive the ball. In fact, larger playing areas benefit teams to keep ball possession, due to more available space to perform tactical individual actions without pressing from opponents. $^{21,22}$ These results highlight the playing area as a key constraint to be considered when designing ball possession drills for tactical individual development. 


\section{Cooperation-based perspective}

Ball possession SSGs induce an increase in the intensity of the exercise and the number of tactical individual actions, as players need to constantly create passing lines or block opponents, thereby increasing the number of movements. ${ }^{18}$ However, the task physical demands and individual actions seem to be constrained by the number of teammates and the playing area dimension. Accordingly, higher physical demands were identified when performing in larger playing areas, especially, in formats of lower cooperation $(4 \mathrm{v} 2+1)$. Under such scenarios, players may have fewer opportunities to maintain ball possession due to a higher pressure from the opponents, which seems to be confirmed by the lower individual participation in these conditions. As consequence, players may have spent most of the time on task attempting to recover the ball, contributing to an increase of the physical demands, and consequently decrease of the tactical individual actions. In contrast, increasing the number of teammates $(4 \mathrm{v} 2+3)$ decreased the physical demands, as less intense running may be required.

In fact, when playing with a numerical advantage and in larger areas, players are less restricted and can decide to recover the ball quickly by pressing the opponent as one of the extra players can perform the balance or the covering tasks. ${ }^{3}$ Bekris, Sambanis ${ }^{40}$ and Praça, Bredt ${ }^{41}$ equally revealed that the use of superiority in SSGs allows players to spend less time at higher intensities compared to balanced formats, indicating a reduction in physical effort. This higher level of cooperation may be able to promote easier team organisation by a better understanding of each individual role, and less exploratory solutions, decreasing the physical demands. ${ }^{27,36}$ Based on these results, using larger areas with a lower level of cooperation $(4 \mathrm{v} 2+1)$ may be used to increase intensity of the task and develop the aerobic and anaerobic systems and recovery abilities. ${ }^{37}$ In contrast, the same area with higher level of cooperation $(4 \mathrm{v} 2+3)$ can be used to reduce pace during periods in which lower, but still demanding, training intensity is desired. Of note, it was previously showed that SSGs with a higher level of cooperation require players to perceive and adapt their behaviour to the context and area of play, decreasing the perceptual and physical demands compared to balanced SSGs. ${ }^{41}$

Also, a max passing speed was promoted on the $\mathrm{L}$ playing area, demonstrating the importance of larger playing areas to develop speed of play, especially if the goal is to stress the transitions. Players seem to show a more exploratory behavior when playing in ball possession at a numerical disadvantage and in smaller playing areas, a situation that seems to force players to vary more their game. A numerical advantage with larger playing areas seem to produce a less exploratory and less varied game, allowing players to perform quickly passing actions. These easier game situations in larger areas can promote more regular and less varied plays, but with quicker passes, while more difficult scenarios force players to explore the varieties of tactical-technical actions they can perform. ${ }^{36}$ Larger playing areas with a lower level of opposition may then constrain players to develop faster ball circulation and it can be used to strengthen offensive and defensive transitions.

\section{Future research}

Surprisingly, RPE scores do not follow the results of the external load variables and, for both oppositionand cooperation-based perspectives, $\mathrm{M}$ playing area promoted a higher perceived exertion for all game formats analysed. Although Casamichana, Castellano ${ }^{42}$ found significant correlations between RPE and most of the external indicators studied, no research has focused on unbalanced formats in ball possession games. More research is then needed to understand the relation of the external workload with the internal load of perceptions during the practice of ball possession unbalanced football games and its relationship with playing area dimension.

Also, this investigation was only focused on ballpossession games; more research is needed to understand the effects of the playing area dimension while playing different unbalance SSGs with the use of regular goals and goalkeeper, and with mini-goals. As a limitation to this investigation, participants from this study knew the time and number of bouts of each SSG, so some behaviours may have been adopted in relation to the remaining time or number of bouts, like delaying or pressing to save energy. Future research would benefit from studying the individual and team behaviours in ball possession tasks without knowing the available time for each format. Another noteworthy investigation would be to analyse the effects of the playing area dimension during the practice of ball possession unbalanced SSGs for different age-group football players. The results from this study may benefit academy coaches to plan development programs according to individuals' age specific needs.

Overall, different playing area dimensions affect player's and team's performance while practicing unbalance football SSGs. To optimise players' technical and tactical skills, coaches are then challenged to design training sessions with representative learning tasks, such as unbalanced SSGs. In addition, it is necessary to adapt the complexity of the task to the players' skill level to maximise their perceptual, visual and attention skills. ${ }^{43}$ 


\section{Conclusion}

From a practical point of view, and through an opposition-based perspective, Balanced formats $(4 \mathrm{v} 4)$ promote more individual and team variability, while SSGs in larger playing areas against more opponents (4v5) encourage the use of intense running. From a cooperation-based perspective, larger playing areas and a higher level of cooperation $(4 \mathrm{v} 2+3)$ may foster technical development, and larger playing areas with a low level of cooperation $(4 \mathrm{v} 2+1)$ promote the development of high-intensity running. Alongside, S playing area, especially in formats of lower number, allow players to walk more distances and decrease the pace of the game.

Summarising, larger playing areas and formats with players in inferiority may be used to increase intensity of the exercise and to promote the development of tactical individual actions of passing, and it can be implemented to develop offensive and defensive transitions, as it allows improving ball circulation speed. These formats may be utilised two days before the match, to develop speed and max passing speed. On the other hand, formats with teams in superiority and in smaller playing areas can be used in recovery sessions, and to develop offensive and defensive organisation, under pressure. These last formats may be practiced in the first two days after the official match to allow an active recovery under real game scenarios, and to develop specific football strength and muscular endurance to substitute players.

Based on the results from this study, coaches should carefully consider the number of opponents/teammates and the playing area dimension when designing sessions during the weekly microcycle, as it greatly affects the players' physical workload and tactical individual actions.

\section{Authors' Note}

Diogo Coutinho is also affiliated with Department of Sports Sciences, Exercise and Health, University of Trás-os-Montes and Alto Douro, Vila Real, Portugal and University Institute of Maia, ISMAI, Maia, Portugal.

\section{Authors' contributions}

NAN and BT contributed to all. BG and DC contributed to literature review, data analysis and interpretation, statistical analyses and reviewing/editing a draft of the manuscript. FYN contributed to literature review, data analysis and interpretation and reviewing/editing a draft of the manuscript.

\section{Declaration of Conflicting Interests}

The author(s) declared no potential conflicts of interest with respect to the research, authorship, and/or publication of this article.

\section{Funding}

The author(s) declared the following potential conflicts of interest with respect to the research, authorship, and/or publication of this article: This research was partially supported by Portuguese Foundation for Science and Technology, I.P., grant number UID04045/2020, Portugal.

\section{ORCID iDs}

Nuno André Nunes (D) https://orcid.org/0000-0002-23214998

Bruno Travassos (D) https://orcid.org/0000-0002-2165-2687

\section{References}

1. Davids K, Araújo D, Correia V, et al. How small-sided and conditioned games enhance acquisition of movement and decision-making skills. Exerc Sport Sci Rev 2013; 41: 154-161.

2. Davids K, Araújo D and Shuttleworth R. Applications of dynamical system theory to football. In: Science and football V. 2005, pp.537-550. London: Routledge.

3. Travassos B, Vilar L, Araújo D, et al. Tactical performance changes with equal vs unequal numbers of players in small-sided football games. Int J Perform Anal Sport 2014; 14: 594-606.

4. Ibáñez SJ, Pérez-Goye E, García-Rubio J, et al. Effects of task constraints on training workload in elite women's soccer. Int J Sports Sci Coa 2019; 15: 99-107.

5. Ometto L, Vasconcellos FVA, Cunha FA, et al. How manipulating task constraints in small-sided and conditioned games shapes emergence of individual and collective tactical behaviours in football: a systematic review. Int J Sports Sci Coa 2018; 13(6): 1200-1214.

6. Rabano-Munoz A, Asian-Clemente J, Saez de Villarreal E, et al. Age-related differences in the physical and physiological demands during small-sided games with floaters. Sports (Basel) 2019; 7: 79.

7. Torres-Ronda L, Gonçalves B, Marcelino R, et al. Heart rate, time-motion, and body impacts when changing the number of teammates and opponents in soccer smallsided games. J Strength Cond Res 2015; 29: 2723-2730.

8. Hill-Haas S, Coutts AJ, Dawson BT, et al. Time-motion characteristics and physiological responses of small-sided games in elite youth players: the influence of player number and rule changes. J Strength Cond Res 2010; 24: 2149-2156.

9. Vilar L, Araújo D, Davids K, et al. Science of winning soccer: emergent pattern-forming dynamics in association football. J Syst Sci Complex 2013; 26: 73-84.

10. Sampaio J, Lago C, Gonçalves B, et al. Effects of pacing, status and unbalance in time motion variables, heart rate and tactical behaviour when playing 5-a-side football small-sided games. J Sci Med Sport 2014; 17: 229-233.

11. Sangnier S, Cotte T, Brachet $\mathrm{O}$, et al. Planning training workload in football using small-sided games' density. J Strength Cond Res 2019; 33: 2801-2811. 
12. Halouani J, Chtourou H, Gabbett T, et al. Small-Sided games in team sports training: a brief review. J Strength Cond Res 2014; 28: 3594-3618.

13. Sarmento H, Clemente FM, Harper LD, et al. Small sided games in soccer - a systematic review. Int $J$ Perform Anal Sport 2018; 18: 693-749.

14. Silva P, Travassos B, Vilar L, et al. Numerical relations and skill level constrain co-adaptive behaviors of agents in sports teams. PLoS One 2014; 9: e107112.

15. Sgrò F, Bracco S, Pignato S, et al. Small-sided games and technical skills in soccer training: systematic review and implications for sport and physical education practitioners. J Sports Sci 2018; 6.

16. Gaudino P, Alberti G and Iaia FM. Estimated metabolic and mechanical demands during different small-sided games in elite soccer players. Hum Mov Sci 2014; 36C: 123-133.

17. Martín-García A, Castellano J, Mendez-Villanueva A, et al. Physical demands of ball possession games in relation to the most demanding passages of a competitive match. J Sports Sci Med 2020; 19: 1-9.

18. Rebelo A, Brito J, Fernandes L, et al. Physiological, technical and time-motion responses to goal scoring versus ball possession in soccer small-sided games. Rpcd 2011; 11: 13-20.

19. Castellano J, Casamichana D and Dellal A. Influence of game format and number of players on heart rate responses and physical demands in small-sided games. $J$ Strength Cond Res 2013; 27: 1295-1303.

20. Kalapotharakos VI, Douda H, Spassis A, et al. Heart rate responses during small-sided games. Soccer J 2011; 46- 49.

21. Vilar L, Duarte R, Silva P, et al. The influence of pitch dimensions on performance during small-sided and conditioned soccer games. J Sports Sci 2014; 32: 1751-1759.

22. Vilar L, Esteves PT, Travassos B, et al. Varying numbers of players in small-sided soccer games modifies action opportunities during training. Int J Sports Sci Coa 2014; 9: 1007-1018.

23. Abrantes CI, Nunes MI, Maçãs VM, et al. Effects of the number of players and games type constraints on heart rate, rating of perceived exertion, and technical actions of Small-Sided soccer games. J Strength Cond Res 2012; 26: 976-981.

24. Aguiar M, Botelho G, Gonçalves B, et al. Physiological responses and activity profiles of football small-sided games. J Strength Cond Res 2013; 27: 1287-1294.

25. Owen AL, Twist C and Ford P. Small-sided games: the physiological and technical effect of altering pitch size and player numbers. Insight 2004; 7: 50-53.

26. Williams K and Owen A. The impact of player numbers on the physiological responses to small sided games. $J$ Sports Sci Med 2007; 6: 99-102.

27. Gonçalves B, Marcelino R, Torres-Ronda L, et al. Effects of emphasising opposition and cooperation on collective movement behaviour during football smallsided games. J Sports Sci 2016; 34: 1346-1354.

28. Sasaki T, Okura K, Shibata K, et al. Examination of absolute reliability of mileage measurement using ZEPP play soccer ${ }^{\circledR}$. In: 53rd annual meeting of the Japanese Physical Therapy Association, Japan, 20 August 2019.

29. Aroganam G, Manivannan N and Harrison D. Review on wearable technology sensors used in consumer sport applications. Sensors 2019; 19.

30. Owen AL, Wong DP, Paul D, et al. Physical and technical comparisons between various-sided games within professional soccer. Int J Sports Med 2013; 35: 286-292.

31. Coutts AJ, Rampinini E, Marcora SM, et al. Heart rate and blood lactate correlates of perceived exertion during small-sided soccer games. J Sci Med Sport 2009; 12: 79-84.

32. jamovi. The jamovi project. 1.2 ed. Sydney, Australia, 2020.

33. Cumming G. Understanding the new statistics: effect sizes, confidence intervals, and meta-analysis. New York: Routledge, 2012.

34. Cohen J. Statistical power analysis for the behavioral sciences. 2nd ed. Hillsdale, N.J.: L. Erlbaum Associates: Routledge, 1988.

35. Olthof SBH, Frencken WGP and Lemmink KAPM. Match-derived relative pitch area changes the physical and team tactical performance of elite soccer players in small-sided soccer games. J Sports Sci 2018; 36: 1557-1563.

36. Torrents C, Ric A, Hristovski R, et al. Emergence of exploratory, technical and tactical behavior in smallsided soccer games when manipulating the number of teammates and opponents. PLoS One 2016; 11: e0168866.

37. Clemente FM, Martins FML and Mendes RS. Developing aerobic and anaerobic fitness using smallsided soccer games: methodological proposals. Strength Cond J 2014; 36: 76-87.

38. Castagna C, Impellizzeri FM, Chaouachi A, et al. Effect of training intensity distribution on aerobic fitness variables in elite soccer players: a case study. J Strength Cond Res 2011; 25: 66-71.

39. Dalen T, Sandmæl S, Stevens TGA, et al. Differences in acceleration and high-intensity activities between smallsided games and peak periods of official matches in elite soccer players. J Strength Cond Res. Epub ahead of print 6 February 2019. DOI: 10.1519/JSC.0000000000003081.

40. Bekris E, Sambanis M, Milonys E, et al. The physiological and technical-tactical effects of an additional soccer player's participation in small sided games training. Phys Training 2012; 11.

41. Praça GM, Bredt SGT, Torres JO, et al. Influence of numerical superiority and players' tactical knowledge on perceived exertion and physical and physiological demands in soccer small-sided games. Rev de Psicol Del Deporte 2018; 27: 31-31-38.

42. Casamichana D, Castellano $\mathbf{J}$ and Dellal A. Influence of different training regimes on physical and physiological demands during small-sided soccer games: continuous vs. intermittent format. J Strength Cond Res 2013; 27: 690-697.

43. Praxedes A, Moreno A, Gil-Arias A, et al. The effect of small-sided games with different levels of opposition on the tactical behaviour of young footballers with different levels of sport expertise. PLoS One 2018; 13: e0190157. 